PROCEEDINGS OF THE

AMERICAN MATHEMATICAL SOCIETY

Volume 129, Number 9, Pages 2571-2579

S 0002-9939(01)05940-8

Article electronically published on February 9, 2001

\title{
A REMARK ON A THEOREM OF J. TITS
}

\author{
CURTIS D. BENNETT AND SERGEY SHPECTOROV
}

(Communicated by Stephen D. Smith)

\begin{abstract}
Let $G$ be a rank two Chevalley group and $\Gamma$ be the corresponding Moufang polygon. J. Tits proved that $G$ is the universal completion of the amalgam formed by three subgroups of $G$ : the stabilizer $P_{1}$ of a point $a$ of $\Gamma$, the stabilizer $P_{2}$ of a line $\ell$ incident with $a$, and the stabilizer $N$ of an apartment $A$ passing through $a$ and $\ell$. We prove a slightly stronger result, in which the exact structure of $N$ is not required. Our result can be used in conjunction with the "weak $B N$-pair" theorem of Delgado and Stellmacher in order to identify subgroups of finite groups generated by minimal parabolics.
\end{abstract}

\section{INTRODUCTION}

In the continuing efforts to put together a complete and "simpler" proof of the classification of finite simple groups the problem of identifying the known simple groups from certain of their subgroups remains important. In particular, in one of the cases (the characteristic- $p$-type case) a simple Lie type group $G$ in characteristic $p$ must be reconstructed from its $p$-local subgroups. In the case where the rank of $G$ is at least 3 there seems to be a clear "best" way to do this. Namely, $G$ is known to be the universal closure of the amalgam of its maximal parabolic subgroups. There exists an easy geometric argument for this important fact, based on the simple connectedness of the building on which $G$ acts (cf. Tits [T]).

When the rank of $G$ is two, the universal closure of the amalgam of the maximal parabolics, $\left\{P_{1}, P_{2}\right\}$, is the free amalgamated product of $P_{1}$ and $P_{2}$, and hence infinite. The day is saved by a theorem of Tits ([S], Chapter II, Theorem 8) stating that $G$ is the universal closure of the amalgam of $P_{1}, P_{2}$ and $N$, where $N$ is the normalizer of a Cartan subgroup $C$ from $B:=P_{1} \cap P_{2}$. (This definition does not work when the field is small. A better definition (see below) that works in all cases can be given in terms of the generalized quadrangle on which $G$ acts.) The first proof of this result used the $B N$-pair technique; more recently an elementary proof was found based again on the simple connectedness of the rank three geometry of points, lines, and apartments of the generalized $n$-gon on which $G$ acts.

Suppose we have an injection $\phi$ of the amalgam $\left\{P_{1}, P_{2}\right\}$ into a group $H$. (We will use the bar notation for the images under this injection.) This amounts to finding in $H$ a pair of subgroups $\left\{\bar{P}_{1}, \bar{P}_{2}\right\}$ forming an amalgam isomorphic to $\left\{P_{1}, P_{2}\right\}$. In many cases the subgroups $\bar{P}_{1}$ and $\bar{P}_{2}$ are readily available due to the classification by Delgado and Stellmacher [DGS] of "weak $B N$-pairs". It seems desirable to

Received by the editors January 24, 2000.

1991 Mathematics Subject Classification. Primary 20E42, 20D06, 51E12, 51E24.

The second author received partial support from NSF grant \#9896154. 
complement the theorem of Delgado and Stellmacher by a result showing that under some general conditions $\bar{P}_{1}$ and $\bar{P}_{2}$ generate in $H$ a subgroup isomorphic to $G$. This amounts to extending $\phi$ to an injection from $G$ into $H$. In fact, by Tits' result, for this it suffices to extend $\phi$ to $N$.

Let $N_{i}:=N \cap P_{i}, i=1,2$. If we want to extend $\phi$ to an injection of $\left\{P_{1}, P_{2}, N\right\}$ into $H$, we need to define the image $\bar{N}$ of $N$ as $\left\langle\bar{N}_{1}, \bar{N}_{2}\right\rangle$. Since $N / C \cong D_{2 n}$ (the images of $N_{1}$ and $N_{2}$ in $N / C$ being groups of order 2), to extend $\phi$ we just need to verify the relation $\left(\bar{n}_{1} \bar{n}_{2}\right)^{n}=\bar{c}$, where $n_{i}, i=1,2$, is chosen in $N_{i} \backslash C$ and $c:=\left(n_{1} n_{2}\right)^{n}$.

Since $C \unlhd N$, the groups $\bar{N}_{1}$ and $\bar{N}_{2}$ normalize $\bar{C}$ and act on it. From this we can conclude that $x:=\left(\bar{n}_{1} \bar{n}_{2}\right)^{n}$ must be in $C_{H}(\bar{C})$. Furthermore, if $n_{1}$ and $n_{2}$ are involutions, then, of course, $\bar{n}_{1}$ and $\bar{n}_{2}$ are involutions, too, and hence, they invert $x$. But these are, essentially, all the conditions on $x$ that we have. In particular, even if we know that $x$ is in $\bar{C}$, we may still have two or more candidates for $x$. For example, if $n=4$ and $q$ is odd, there are at least two elements in $\bar{C}$ inverted by $\bar{n}_{1}$ and $\bar{n}_{2}$ (again assuming that $\bar{n}_{1}$ and $\bar{n}_{2}$ are involutions). Setting $x$ equal to any of those candidate elements, we obtain a valid amalgam of three groups regardless of whether $x$ was chosen as the "correct" $\bar{c}$ or not. Of course, we should expect that the wrong choices for $x$ never show up in actual groups, but it appears that we need a non-local condition to see that the completion of the "wrong" amalgam collapses.

The above discussion demonstrates possible complications in the application of Tits' result. The main goal of the present paper is to prove a slightly enhanced version of Tits' theorem, nullifying some of these concerns.

For expository purposes we assumed above that $G$ was simple. In actual applications it is beneficial to also allow almost simple target groups $G$. The exact condition is as follows. Let $\Gamma$ be a finite classical Moufang polygon (see [vM] for a detailed account of generalized polygons), and let $G$ be a group of type-preserving automorphisms of $\Gamma$, containing all point- and line-elations. That is, $G$ contains the so-called little projective group of $\Gamma$ (cf. [vM], Chapter 4). The latter condition only means that $G$ cannot be one of the three exceptional groups: $S p(4,2)^{\prime} \cong A_{6}$, $G_{2}(2)^{\prime} \cong U_{3}(3)$, and the Tits simple group ${ }^{2} F_{4}(2)^{\prime}$. Other than these exceptions, $G$ is, thus, any simple group of Lie type of rank 2 extended by an arbitrary combination of diagonal and field automorphisms. As above, we let $P_{1}$ and $P_{2}$ be two maximal parabolics of $G$ containing a common Borel subgroup. In terms of the polygon $\Gamma, P_{1}$ and $P_{2}$ are the stabilizers in $G$ of a point $a$ and a line $L$ of $\Gamma$, where $a$ and $L$ are incident. Let $N$ be the setwise stabilizer of an apartment containing $a$ and $L$, and let $C \unlhd N$ be the elementwise stabilizer of the same apartment. We further set $N_{i}:=N \cap P_{i}, i=1,2$.

As before, let $\phi$ be an injective morphism of the amalgam $\left\{P_{1}, P_{2}\right\}$ into a group $H$. We will continue using the bar notation for the images under $\phi$.

We can now state our results.

Theorem 1. Under the above setup, if $\left\langle\bar{N}_{1}, \bar{N}_{2}\right\rangle / \bar{C}$ is isomorphic to $D_{2 k}, k \leq n$, then $k=n, \phi$ extends to an injection of the rank three amalgam $\left\{P_{1}, P_{2}, N\right\}$ into $H$, and $\bar{G}:=\left\langle\bar{P}_{1}, \bar{P}_{2}\right\rangle$ is isomorphic to $G$.

Let $G_{0}$ be the intersection of $G$ with the (normal) subgroup of Aut $\Gamma$ generated by all elations and generalized homologies. Let $C_{0}:=C \cap G_{0}$, a Cartan subgroup in $G$. As a corollary to Theorem 1 we also obtain the following. 
Theorem 2. If $\bar{C}_{0}$ is self-centralized in $H$, then $\bar{G}$ is isomorphic to $G$.

It may appear from the phrasing of the statement of Theorem 1 that the third claim in it, that $\bar{G}$ is isomorphic to $G$, is obtained as a corollary of the other two via an application of Tits' theorem. In reality, we bypass Tits and show directly, using only a nice lemma of Delgado and Stellmacher, that $\phi$ extends to an isomorphism of the entire group $G$ onto its image $\bar{G}$ in $H$.

We conclude the paper by discussing the exceptional groups $S p(4,2)^{\prime}, G_{2}(2)^{\prime}$ and ${ }^{2} F_{4}(2)^{\prime}$. Are they true exceptions from Theorem 11, or is it merely that our proof does not work well for these groups? First of all, in all the three groups $C=1$. Thus, the claim as in Tits' theorem simply coincides for these groups with the subcase $k=n$ of Theorem [1. When $G=S p(4,2)^{\prime}$ or $G_{2}(2)^{\prime}$, and $k=n$, we show that the universal closure of the amalgam $\left\{P_{1}, P_{2}, N\right\}$ is infinite. Therefore, they are true exceptions from both our Theorem [1 and the original Tits' theorem. The situation is different for $G={ }^{2} F_{4}(2)^{\prime}$, the Tits simple group. Namely, we prove the following.

Theorem 3. Under the setup described before Theorem 11, if $G={ }^{2} F_{4}(2)^{\prime}$, then the universal closure of the amalgam $\left\{P_{1}, P_{2}, N\right\}$ coincides with $G$. Furthermore, if $\bar{N}:=\left\langle\bar{N}_{1}, \bar{N}_{2}\right\rangle \cong D_{2 k}$, where $k \leq 8$, then $k=8$ and $\bar{G}:=\left\langle\bar{P}_{1}, \bar{P}_{2}\right\rangle$ is isomorphic to $G$.

Notice that since here $C=1$ and $n=8$, the second statement in Theorem 3 is analogous to the one in Theorem 1 .

\section{Preliminaries}

In this section $G$ is an arbitrary group generated by its two subgroups $P_{1}$ and $P_{2}$. The coset geometry $\Gamma=\Gamma\left(G, P_{1}, P_{2}\right)$ has as elements all (right) cosets of $P_{1}$ and $P_{2}$ in $G$. A coset $P_{1} a$ is incident to a coset $P_{2} b$ if and only if $P_{1} a \cap P_{2} b \neq \emptyset$. Thus $\Gamma$ is a connected rank 2 geometry and $G$ acts on $\Gamma$ flag-transitively by right shifts. We will assume throughout the section that the geometry $\Gamma$ is thick, that is, every element of $\Gamma$ is incident with at least three other elements. This corresponds to the group-theoretic condition $\left[P_{i}: P_{1} \cap P_{2}\right] \geq 3, i=1,2$.

Frequently, we will view $\Gamma$ as a bipartite graph - the incidence graph of the geometry $\Gamma$. The group $G$ acts transitively on each bipartite half of $\Gamma$, and $x_{i}:=$ $P_{i}, i=1,2$, are representatives of the two orbits of $G$ on the vertex set of $\Gamma$. Furthermore, every edge of $\Gamma$ is conjugate to $\left\{x_{1}, x_{2}\right\}$.

Recall that the action of $G$ on $\Gamma$ is called locally s-path transitive if for every vertex $v$ of $\Gamma$ the stabilizer of $v$ in $G$ acts transitively on the set of all paths of length $s$ (no returns allowed) starting at $v$. Clearly, this property holds for all $v$ once it is checked for $v=x_{1}$ and $x_{2}$.

Lemma 2.1. Suppose the action of $G$ on $\Gamma$ is locally $s$-transitive and that $\Gamma$ has girth $g=2 k$, where $k<s$. Then the diameter of $\Gamma$ is $k$. In particular, $\Gamma$ is a generalized $k$-gon geometry. Furthermore, $s=k+1$.

Proof. It follows from our assumptions that every path in $\Gamma$ of length $s$ is contained in a cycle of length $g=2 k \leq 2(s-1)$. This implies that the diameter $d$ of $\Gamma$ is at most $k$. As $k \leq d$, we conclude that $d=k$. Thus, the geometry $\Gamma$ is a generalized $k$-gon. It remains to see that $s$ is at most $k+1$. However, that is clear because there are two kinds of $k+2$-paths starting from a given vertex $v$ : for one kind the 
distance from $v$ to the end vertex is $k-2$, and for the other it is $k$. Here we use that $\Gamma$ is thick and therefore a vertex at distance $k-1$ is adjacent with at least two vertices at distance $k$.

The above lemma will be used in combination with the following lemma bounding the girth of $\Gamma$.

Suppose $C$ is a subgroup of $P_{1} \cap P_{2}$. Suppose further that for $i=1,2$ we have an element $n_{i} \in P_{i} \backslash\left(P_{1} \cap P_{2}\right)$ such that $n_{i}^{2} \in C$ and $n_{i} \in N_{G}(C)$. Set $t:=n_{1} n_{2}$ and

$$
x_{i}:= \begin{cases}x_{1}^{t^{j}}, & \text { if } i=2 j+1 ; \text { and } \\ x_{2}^{t^{j}}, & \text { if } i=2 j+2 .\end{cases}
$$

Lemma 2.2. The following hold:

(1) $x_{i}$ is adjacent to $x_{i+1}$ and $x_{i} \neq x_{i+2}$ for all $i$; in particular, $\tau:=\left(\ldots, x_{-1}, x_{0}\right.$, $\left.x_{1}, x_{2}, \ldots\right)$ is an infinite path without returns;

(2) $n_{i}, i=1,2$, acts on $\tau$ by reflecting it in $x_{i}$, and $C$ stabilizes $\tau$ elementwise;

(3) if $t^{n} \in C$, then $x_{i}=x_{i+2 n}$ for all $i$; in particular, $\left(x_{0}, x_{1}, \ldots x_{2 n-1}, x_{0}\right)$ is a closed path without returns.

Remark. In (1) and (2) we view $\tau$ as a two-way infinite sequence of vertices, and not as a subset of $\Gamma$. Notice also that the conclusion in (3) implies that the girth of $\Gamma$ does not exceed $2 n$, which is the bound we need.

Proof. If $i=2 j+1$, then $\left(x_{i}, x_{i+1}\right)=\left(x_{1}, x_{2}\right)^{t^{j}}$ and hence $x_{i}$ and $x_{i+1}$ are adjacent. If $i=2 j+2$, then again $x_{i}$ and $x_{i+1}$ are adjacent since $x_{2}$ and $x_{3}$ are adjacent (indeed, $x_{2}=x_{2}^{n_{2}}$ and $\left.x_{3}=x_{1}^{t}=x_{1}^{n_{2}}\right)$ and $\left(x_{i}, x_{i+1}\right)=\left(x_{2}, x_{3}\right)^{t^{j}}$. So $\tau$ is indeed a path and $t$ acts on $\tau$ by shifting it by two edges in the positive direction. Clearly, $\tau$ has no returns since $t$ fixes neither $x_{1}$ nor $x_{2}$. This proves (1).

For (2), observe, first of all, that since $n_{1}$ and $n_{2}$ leave $C$ invariant, so does $t$. Since $C$ fixes both $x_{1}$ and $x_{2}, C$ must fix every vertex $x_{i}$. Next, notice that $\left(n_{2} n_{1}\right)^{j}=c_{j} t^{-j}$ for some $c_{j} \in C$. Therefore, for $j \geq 0$ we have $x_{2 j+1}^{n_{1}}=x_{1}^{t^{j} n_{1}}=$ $x_{1}^{n_{1} c_{j} t^{-j}}=x_{1}^{t^{-j}}=x_{-2 j+1}$. Also, $x_{2 j+2}^{n_{1}}=x_{2}^{t^{j} n_{1}}=x_{2}^{n_{1} c_{j} t^{-j}}=x_{0}^{t^{-j}}=x_{-2 j}$. This proves that $x_{i}^{n_{1}}=x_{-i+2}$ for all $i \geq 1$. As $n_{1}^{2} \in C$, we also have that $x_{-i+2}^{n_{1}}=x_{i}$, that is, the action of $n_{1}$ on $\tau$ is as claimed. Since $n_{2}=n_{1}^{-1} t$ we also have that $n_{2}$ acts on $\tau$ by reflecting it in $x_{2} ;(2)$ is proved.

If $t^{n} \in C$, then $x_{i}=x_{i}^{t^{n}}=x_{i+2 n}$, and (3) follows as well.

\section{ProOFs}

The proof of Theorem 1 will be comprised of Lemmas 3.1 through 3.5 In particular, in all those lemmas we assume the hypotheses of Theorem 1, We will continue using the notation from the introduction. Additionally, let $\hat{G}$ be the free amalgamated product of $P_{1}$ and $P_{2}$. We will use the "anti-hat" notation: $\hat{P}_{i}$ will stand for the obvious preimage of $P_{i}$ in $\hat{G}$; $\hat{g} \in \hat{G}$ will be an element in the preimage of $g \in G$ (usually, $\hat{g}$ will be in some $\hat{P}_{i}$, making it unique). Let $\pi$ denote the canonical surjection of $\hat{G}$ onto $G$. Since $\phi \circ \pi$, where $\pi$ is restricted to $\hat{P}_{1} \cup \hat{P}_{2}$, is an isomorphism from $\left\{\hat{P}_{1}, \hat{P}_{2}\right\}$ to $\left\{\bar{P}_{1}, \bar{P}_{2}\right\}$, it extends to a homomorphism $\psi$ from $\hat{G}$ to $H$ (having $\bar{G}$ as its image). Continuing the trend, we will also use the combined "bar-anti-hat" notation, meaning that normally $\psi(\hat{g})=\bar{g}$. 
To the three groups $G, \bar{G}$, and $\hat{G}$ there correspond three coset geometries:

$$
\begin{aligned}
& \Gamma:=\Gamma\left(G, P_{1}, P_{2}\right), \\
& \bar{\Gamma}:=\Gamma\left(\bar{G}, \bar{P}_{1}, \bar{P}_{2}\right), \\
& \hat{\Gamma}:=\Gamma\left(\hat{G}, \hat{P}_{1}, \hat{P}_{2}\right) .
\end{aligned}
$$

The homomorphisms $\pi$ and $\psi$ induce morphisms of $\hat{\Gamma}$ onto $\Gamma$ and $\bar{\Gamma}$. By abuse of notation we will use the same notation $\pi$ and $\psi$ for these induced morphisms. Notice that $\pi$ and $\psi$ are coverings both of geometries and graphs. Also notice that $\Gamma$ is the Moufang generalized $n$-gon for $G$ and $\hat{\Gamma}$ is a tree.

Let $\hat{x}_{1}$ and $\hat{x}_{2}$ be the cosets $\hat{P}_{1}$ and $\hat{P}_{2}$, respectively. Furthermore, set $x_{i}:=$ $\pi\left(\hat{x}_{i}\right)=P_{i}$ and $\bar{x}_{i}:=\psi\left(\hat{x}_{i}\right)=\bar{P}_{i}, i=1,2$.

Lemma 3.1. The groups $G, \bar{G}$ and $\hat{G}$ act locally $n+1$-path transitively on the corresponding graphs $\Gamma, \bar{\Gamma}$ and $\hat{\Gamma}$.

Proof. For $G$ and $\Gamma$, this is the Tits property for the Moufang generalized polygons. It holds since by assumption $G$ contains all elations. (See $\mathrm{vM}$, Chapter 4.)

Since $\pi$ is a covering, it establishes a bijection between all paths in $\hat{\Gamma}$ starting at $\hat{x}_{i}$ and all paths in $\Gamma$ starting at $x_{i}$. Furthermore, if $\hat{\gamma}$ is a path in $\hat{\Gamma}$ and $\gamma=\pi(\hat{\gamma})$, then, for every $\hat{g} \in \hat{G}, \pi\left(\hat{\gamma}^{\hat{g}}\right)=\gamma^{g}$, where $g=\pi(\hat{g}) \in G$. Thus, the action of $\hat{P}_{i}$ on the paths in $\hat{\Gamma}$ starting at $\hat{x}_{i}$ is equivalent to the action of $P_{i}$ on the paths in $\Gamma$ starting at $x_{i}$. This implies that the action of $\hat{G}$ on $\hat{\Gamma}$ is locally $n+1$-path transitive, since the action of $G$ on $\Gamma$ is. (Notice that every vertex in $\hat{\Gamma}$ is $\hat{G}$-conjugate to either $\hat{x}_{1}$, or $\hat{x}_{2}$.)

Similarly, taking $\psi, \bar{G}$, and $\bar{\Gamma}$ in place of $\pi, G$ and $\Gamma$, respectively, we observe that the action of $\hat{P}_{i}$ on the paths starting at $\hat{x}_{i}$ is equivalent to the action of $\bar{P}_{i}$ on the paths starting at $\bar{x}_{i}$. Hence the action of $\bar{G}$ on $\bar{\Gamma}$ is locally $n+1$-path transitive too.

Choose $\hat{n}_{i} \in \hat{N}_{i} \backslash \hat{C}, i=1,2$. (Clearly, $\hat{N}_{i}$ and $\hat{C}$ are defined as the subgroups of $\hat{P}_{i}$ that $\pi$ maps to $N_{i}$ and $C$, respectively.) Let $\hat{N}:=\left\langle\hat{N}_{1}, \hat{N}_{2}\right\rangle$. Since $\pi$ establishes an isomorphism of $\hat{N}_{i}$ onto $N_{i}$, we have that $\hat{C} \triangleleft \hat{N}_{i}, i=1,2$, and hence $\hat{C} \triangleleft \hat{N}$. Let $n_{i}:=\pi\left(\hat{n}_{i}\right)$ and $\bar{n}_{i}:=\psi\left(\hat{n}_{i}\right)$. Since $\left[N_{i}: C\right]=2$, we have that $\left[\hat{N}_{i}: \hat{C}\right]=2$ and hence $\hat{n}_{i}^{2} \in \hat{C}$. Clearly, also $n_{i}^{2} \in C$ and $\bar{n}^{2} \in \bar{C}$. Define $\hat{t}:=\hat{n}_{1} \hat{n}_{2}$ and

$$
\hat{x}_{i}:= \begin{cases}\hat{x}_{1}^{\hat{t}^{j}}, & \text { if } i=2 j+1 ; \text { and } \\ \hat{x}_{2}^{\hat{t}^{j}}, & \text { if } i=2 j+2\end{cases}
$$

Let $x_{i}:=\pi\left(\hat{x}_{i}\right)$ and $\bar{x}_{i}:=\psi\left(\hat{x}_{i}\right)$ for all $i$. Recall that by assumption $\left\langle\bar{N}_{1}, \bar{N}_{2}\right\rangle / \bar{C} \cong$ $D_{2 k}$. Therefore, $\left(\bar{n}_{1} \bar{n}_{2}\right)^{k} \in \bar{C}$.

Lemma 3.2. The following hold:

(1) $\hat{\tau}:=\left(\ldots, \hat{x}_{-1}, \hat{x}_{0}, \hat{x}_{1}, \hat{x}_{2}, \ldots\right)$ is a path without returns in $\hat{\Gamma} ; \hat{n}_{i}, i=1,2$, acts on $\hat{\tau}$ by reflecting it in $\hat{x}_{i}$ and $\hat{C}$ fixes $\hat{\tau}$ elementwise;

(2) $\left(x_{0}, x_{1}, \ldots, x_{2 n-1}, x_{0}\right)$ is an apartment in $\Gamma$; and

(3) $\bar{x}_{i}=\bar{x}_{i+2 k}$ for all $i$; that is, $\left(\bar{x}_{0}, \bar{x}_{1}, \ldots, \bar{x}_{2 k-1}, \bar{x}_{0}\right)$ is closed path without returns.

Proof. Part (1) follows from Lemma 2.2 parts (1) and (2). Part (2) follows since $\left(x_{0}, x_{1}, \ldots, x_{2 n-1}, x_{0}\right)$ is, clearly, the apartment, of which $N$ is the stabilizer. Finally, part (3) follows from Lemma $2.2(3)$, since $\left(\bar{n}_{1} \bar{n}_{2}\right)^{k} \in \bar{C}$. 
Corollary 3.3. If $\bar{G}$ satisfies the assumptions of Theorem 1 , then $\bar{\Gamma}$ is a generalized $n$-gon, $k=n$ and $\left(\bar{x}_{0}, \bar{x}_{1}, \ldots, \bar{x}_{2 n-1}, \bar{x}_{0}\right)$ is an apartment in $\bar{\Gamma}$.

Proof. In view of Lemma 2.1 the claim follows from Lemmas 3.1 and 3.2 (3).

At this point using that the amalgam $\left(\bar{P}_{1}, \bar{P}_{2}\right)$ is isomorphic to $\left(P_{1}, P_{2}\right)$ we could prove that $\Gamma$ is Moufang and then refer to the classification of Moufang $n$-gons. Instead we will give a direct proof.

Suppose a generalized $n$-gon $\Delta$ is covered by a tree $\hat{\Delta}$. By a track in $\hat{\Delta}$ we mean a connected component in the full preimage of an apartment of $\Delta$. A track is a (doubly-) infinite path folding onto the corresponding apartment. For the family of all tracks in $\hat{\Delta}$ we have the following two properties: the uniqueness property states that every $n+1$-path is contained in a unique track, and the exchange property states that if the distinct $2 n$-paths $\hat{a}_{0} \hat{a}_{1} \ldots \hat{a}_{n} \ldots \hat{a}_{2 n}$ and $\hat{a}_{0} \hat{a}_{1} \ldots \hat{a}_{n} \hat{b}_{n+1} \ldots \hat{b}_{2 n}$ are parts of two tracks, then the path $\hat{a}_{2 n} \hat{a}_{2 n-1} \ldots \hat{a}_{n} \hat{b}_{n+1} \ldots \hat{b}_{2 n}$ is also part of a track (cf. [DGS]). We would like to claim that the family of tracks defines $\Delta$ and the covering $\hat{\Delta} \longrightarrow \Delta$ up to isomorphism. For that we define the following equivalence on the vertices of $\hat{\Delta}$. Two vertices $\hat{a}, \hat{b}$ of $\hat{\Delta}$ are called elementary equivalent if $\hat{a}$ and $\hat{b}$ belong to a common track and are distance $2 n$ apart. Vertices $\hat{a}$ and $\hat{b}$ are called equivalent if they are connected by a number of elementary equivalences.

Lemma 3.4 ([DGS]). Two vertices $\hat{a}$ and $\hat{b}$ of $\hat{\Delta}$ map onto the same vertex of $\Delta$ if and only if they are equivalent in the above sense.

Proof. For the sake of completeness, we will give a proof of this known fact. It is clear that two elementary equivalent vertices map onto the same vertex of $\Delta$ (since vertices at distance $2 n$ along a track map to the same vertex of the $2 n$-cycle in $\Delta$ ). Consequently any two equivalent vertices of $\hat{\Delta}$ must also map to the same vertex of $\Delta$. Conversely, suppose $\hat{a}$ and $\hat{b}$ are two vertices of $\hat{\Delta}$ mapping onto the same vertex of $\Delta$. We will prove that $\hat{a}$ and $\hat{b}$ are equivalent by induction on the distance $m=d(\hat{a}, \hat{b})$ from $\hat{a}$ to $\hat{b}$. Let $\hat{\gamma}$ denote the shortest path from $\hat{a}$ to $\hat{b}$. Then the image of $\hat{\gamma}$ is a closed path in $\Delta$ with no returns (except at the end point). If $0<m<2 n$, then the image of $P$ would be a closed path of length less than $2 n$ contrary to the fact that $\Delta$ has girth $2 n$. Hence if $m<2 n$, then $\hat{a}=\hat{b}$ and, trivially, they are equivalent. Now, suppose $m \geq 2 n$, say, $\hat{\gamma}=\hat{a} \hat{a}_{1} \hat{a}_{2} \ldots \hat{a}_{m-1} \hat{b}$. By the uniqueness condition, the $n+1$-path $\hat{a} \hat{a}_{1} \ldots \hat{a}_{n+1}$ is part of a unique track $\hat{\Sigma}$. Let $\hat{c}$ be the vertex of $\hat{\Sigma}$ at distance $2 n$ from $\hat{a}$ such that the shortest path from $\hat{a}$ to $\hat{c}$ contains the vertices $\hat{a}_{1}, \ldots, \hat{a}_{n+1}$. Then $\hat{c}$ is elementary equivalent to $\hat{a}$, and hence it maps onto the same vertex of $\Delta$, as $\hat{a}$ and $\hat{b}$ do. Moreover, $d(\hat{c}, \hat{b}) \leq m-2$, since $\hat{c}$ is at distance $n-1$ from the vertex $\hat{a}_{n+1}$. Hence, by induction the vertices $\hat{c}$ and $\hat{b}$ are equivalent. This implies, however, that $\hat{a}$ and $\hat{b}$ are also equivalent.

We have now established that the set of tracks of the covering tree determines the generalized $n$-gon.

Lemma 3.5. $\bar{\Gamma}$ is isomorphic to $\Gamma$, and $\bar{G}$ is isomorphic to $G$.

Proof. By Lemma 3.4 it suffices to show that the set of tracks corresponding to the covering $\pi$ coincides with set of tracks corresponding to $\psi$. Indeed, if $\pi$ and $\psi$ produce the same set of tracks in $\hat{\Gamma}$, then, for $\hat{a}, \hat{b} \in \hat{\Gamma}$, we have that $\pi(\hat{a})=\pi(\hat{b})$ if and only if $\psi(\hat{a})=\psi(\hat{b})$. Thus, the map $\phi: \Gamma \longrightarrow \bar{\Gamma}$ defined by $\phi(\pi(\hat{a}))=\psi(\hat{a})$ for 
$\hat{a} \in \hat{\Gamma}$ is a well-defined bijection between $\Gamma$ and $\bar{\Gamma}$, and clearly it is an isomorphism of geometries corresponding to our isomorphism $\phi$ of $\left\{P_{1}, P_{2}\right\}$ onto $\left\{\bar{P}_{1}, \bar{P}_{2}\right\}$.

Notice that by Lemma $3.3 \bar{\tau}$ is an apartment in $\bar{\Gamma}$. Therefore, $\hat{\tau}$ is a track with regard to both $\pi$ and $\psi$. Since $\hat{G}$ acts on $\hat{\Gamma}$ locally $n+1$-transitively, the uniqueness condition for tracks implies that both sets of tracks coincide with $\left\{\hat{\tau}^{\hat{G}}\right\}$.

This completes the proof of Theorem[1. As the following lemma shows, Theorem 2 is an immediate corollary of Theorem 1 .

Lemma 3.6. If $\bar{C}_{0}$ is self-centralized in $H$, then $\left\langle\bar{N}_{1}, \bar{N}_{2}\right\rangle / \bar{C} \cong D_{2 k}$ for some $k \leq$ $n$.

Proof. Notice that $C_{0}$ is a Cartan subgroup in $G_{0}$ and hence it is abelian. Since, by assumption, $G_{0}$ contains all elations of $\Gamma$, without loss of generality we may assume that $n_{i}=\pi\left(\hat{n}_{i}\right)$ is contained in $N_{i} \cap G_{0}, i=1,2$. Then $c:=\left(n_{1} n_{2}\right)^{n}$ (which is contained in $C$ by assumption) is contained in $C_{0}$ and hence it centralizes $C_{0}$. Now let $\hat{c}:=\left(\hat{n}_{1} \hat{n}_{2}\right)^{n}$. (Clearly, $\pi(\hat{c})=c$.) Observe that $\hat{C}$ is normal in both $\hat{N}_{i}$ and therefore $\left[\hat{c}, \hat{C}_{0}\right]$ (where $\hat{C}_{0}$ is, naturally, the preimage of $C_{0}$ in $\hat{C}$ ) is contained in $\hat{C}$. Since $\pi$ establishes an isomorphism between $\hat{C}$ and $C$, and since $\pi\left(\left[\hat{c}, \hat{C}_{0}\right]\right)=\left[c, C_{0}\right]=1$, we must also have that $\hat{c}$ centralizes $\hat{C}_{0}$.

We now pass to the group $H$. Define $\bar{n}_{i}:=\phi\left(n_{i}\right)=\psi\left(\hat{n}_{i}\right), \bar{c}:=\left(\bar{n}_{1} \bar{n}_{2}\right)^{n}=\psi(\hat{c})$. Notice that $\bar{C}_{0}=\phi\left(C_{0}\right)=\psi\left(\hat{C}_{0}\right)$. Therefore, $\left[\bar{c}, \bar{C}_{0}\right]=\psi\left(\left[\hat{c}, \hat{C}_{0}\right]\right)=\psi(1)=1$. Since $\bar{C}_{0}$ is, by assumption, self-centralized, we conclude that $\bar{c} \in \bar{C}_{0} \leq \bar{C}$. This proves that $\left\langle\bar{n}_{1}, \bar{n}_{2}\right\rangle / \bar{C} \cong D_{2 k}$ for some $k$ dividing $n$.

We now turn to the exceptional groups $S p(4,2)^{\prime}, G_{2}(2)^{\prime}$ and ${ }^{2} F_{4}(2)^{\prime}$. In this case, for $i=1$ or 2 (or both) we have $N_{i}=N \cap P_{i}=C=1$. To see this, note that, as we are working over $G F(2)$, if $g \in G$ stabilizes an apartment $A$ of $\Gamma$ and a point or line $x \in A$, then $g$ fixes the unique element $y \notin A$ incident with $x$. Consequently, $g$ fixes the half-apartment containing $x, y$, and the element $x^{\prime}$ opposite to $x$ in $A$, and it follows that $g$ is an elation. According to Theorem 4.8.7 from vM] (quoted from $[\mathrm{Sz}]$ ), however, if $G=S p(4,2)^{\prime}$ or $G_{2}(2)^{\prime}$, then $G$ does not contain at least one of the two classes of elations. Thus, for $i=1$ or 2 , we have $N_{i}=N \cap P_{i}=C=1$. This immediately implies that the universal group of the amalgam $\left\{P_{1}, P_{2}, N\right\}$ is isomorphic to the free amalgamated product of $P_{i}$ with the universal completion of the amalgam $\left\{P_{3-i}, N\right\}$, which is, in turn, isomorphic to the free amalgamated product of the latter two groups. Thus, the universal completion of $\left\{P_{1}, P_{2}, N\right\}$ is infinite. Consider now the case $G={ }^{2} F_{4}(2)^{\prime}$, so that $\Gamma$ is the corresponding Moufang generalized octagon $(n=8)$ of order $(2,4)$. (We distinguish points and lines of $\Gamma$ by the condition that every point is on 5 lines and every line has three points.) Theorem 4.8.7 from [vM] tells us that in this case $G$ contains all elations of order 2. In particular, $G$ contains an entire apartment stabilizer from Aut $G={ }^{2} F_{4}(2)$. That is, $N \cong D_{16}$ and both $N_{1}$ and $N_{2}$ are of order 2. Notice that $G$ does not contain the (point) elations of order 4 .

Lemma 3.7. $G$ acts on $\Gamma$ locally 7-, but not 8-path transitively; also, $G$ acts transitivily on 8-paths starting from a point.

Proof. Suppose $G$ is locally $s$-, but not $s+1$-path transitive.

Let $\tilde{G}={ }^{2} F_{4}(2)$, so that $[\tilde{G}: G]=2$. The group $\tilde{G}$ acts on $\Gamma$ and this action is locally 9-path transitive. Let $\left\{x_{1}, x_{2}\right\}$ be an edge of $\Gamma$. Let $\tilde{B}$ be the stabilizer 
of this edge in $\tilde{G}$, i.e., $\tilde{B}$ is a Borel subgroup in $\tilde{G}$. It is well known that $\tilde{B}$ acts regularly on the set of 9-paths starting from $x_{1}$ and $x_{2}$. Since $B:=\tilde{B} \cap G$ has index two in $\tilde{B}$, we have that $B$ has exactly two orbits on those 9-paths. Consequently, $G$ has exactly two orbits on 9-paths starting with each particular type of element (point or line).

We have shown that $s \leq 8$. If $s=8$, then, in particular, $G$ is transitive on 8-paths starting from (and ending at) a line. Since the line elations are involutions, they are all contained in $G$. Hence the stabilizer of an 8-path $\gamma$ starting at a line interchanges the two 9-paths extending $\gamma$. However, that means that $G$ is transitive on 9-paths starting from a point, a contradiction. Thus, $s \leq 7$.

It remains to show that $G$ is transitive on 8-paths starting from a point. (This assures, in particular, that $s \geq 7$.) Suppose $G$ is not transitive on the set of all those 8-paths, and suppose $\gamma$ is one of them. The stabilizer of $\gamma$ in $\tilde{G}$ is a subgroup $Z_{4}$ of point elations. This subgroup meets $G$ in just a $Z_{2}$, that is, the stabilizer of $\gamma$ in $G$ has two orbits on the four 9-paths extending $\gamma$. However, this means that the number of $G$-orbits on the set of 9-paths starting from a point is at least four, a contradiction.

Since $G$ is not locally $n+1$ - (that is, 9-) path transitive, our generic argument does not work, so we need to use a different technique. We first do the Tits' case.

Lemma 3.8. $G$ is the universal completion of the amalgam $\left\{P_{1}, P_{2}, N\right\}$.

Proof. Let $A$ be the apartment in the generalized octagon $\Gamma$, passing through $x_{1}$ and $x_{2}$, that is stabilized by $N$. Consider a rank three geometry $\Theta$, whose elements are the elements of the generazed octagon $\Gamma$, together with all the apartments $G$ conjugate to $A$. (Since $G$ has index 2 in the full automorphism group of $\Gamma, G$ has two orbits of equal size on the set of apartments; we simply take one of the two orbits.) The incidence between elements of $\Gamma$ is natural, as in $\Gamma$. The incidence between the elements of $\Gamma$ and the apartments is by inclusion.

We first observe that $\Theta$ is a strongly connected geometry, that is, the residue of every element of $\Theta$ is connected. Indeed, clearly, the residue of an apartment is connected. Also, if $a$ is an element of $\Gamma$ (say, a point), then, by simple counting, $a$ and any two lines in the residue of $a$ are contained in exactly $2^{10}$ apartments; exactly half of those apartments are elements of $\Theta$. (The latter claim holds since $G$ is transitive on 2-paths starting from a line, and since the two $G$-orbits on apartments have equal size.) Similarly, the residue of every line is connected.

We also observe that $G$ acts flag-transitively on $\Theta$ and that $\left\{P_{1}, P_{2}, N\right\}$ is the amalgam of maximal parabolics corresponding to the maximal flag $\left\{x_{1}, x_{2}, A\right\}$. By T], $G$ is the universal closure of $\left\{P_{1}, P_{2}, N\right\}$ if and only if $\Theta$ is simply connected. Thus, we need to show that $\pi_{1}(\Theta)=1$. Viewing $\Gamma$ as a subset of $\Theta$ and choosing the base point $v$ as a point of $\Gamma$, we observe that every closed path in $\Theta$, starting and ending at $v$, is homotopic to a closed path in $\Gamma$. Furthermore, it is well known that every cycle in $\Gamma$ is homotopic to a product of apartments. Hence the lemma will follow if we show that every apartment in $\Gamma$ can be decomposed as a product of apartments conjugate to $A$. (The apartment $A$ and its conjugates are contractible in $\Theta$ as they are contained in the residue of an element of $\Theta$.) Suppose $B=$ $\left(v_{0}, v_{1}, \ldots, v_{15}, v_{0}\right)$ is an apartment not conjugate to $A$. Without loss of generality, $v_{0}$ is a point. Let $\gamma_{1}, \ldots, \gamma_{5}$ be the five 8-paths joining $v_{0}$ with $v_{8}$. Without loss of generality, $\gamma_{1}=\left(v_{0}, v_{1}, \ldots, v_{8}\right)$ and $\gamma_{2}=\left(v_{0}, v_{15}, v_{14}, \ldots, v_{8}\right)$. Define a graph $\Sigma$, 
whose vertices are the five paths $\gamma_{i}$ and where $\gamma_{i}$ is adjacent to $\gamma_{j}, i \neq j$, if and only if $\gamma_{i} \gamma_{j}^{-1}$ is an apartment conjugate to $A$. By Lemma 3.7. exactly two (out of four) apartments containing $\gamma_{i}$ are conjugate to $A$, which means that $\Sigma$ has valency 2. Therefore, $\Sigma$ is isomorphic to a 5 -cycle. The vertices $\gamma_{1}$ and $\gamma_{2}$ are not adjacent, but in a 5 -cycle they must have a common neighbour $\gamma_{i}$. However, that means that $B=\gamma_{1} \gamma_{2}^{-1}=\left(\gamma_{1} \gamma_{i}^{-1}\right)\left(\gamma_{i} \gamma_{2}^{-1}\right)$ is contractible.

To complete the proof of Theorem 3 it remains to show that $k=8$. Suppose $k<8$. Define $\bar{\Gamma}$ as in the beginning of this section. By Lemma 3.7 $G$ is locally 7 path transitive on $\Gamma$ and it is transitive on 8-paths starting from a point. Repeating the argument from Lemma 3.1, we obtain that $\bar{G}$, too, is locally 7 -path transitive on $\bar{\Gamma}$ and it is transitive on 8-paths in $\bar{\Gamma}$ starting from a point. (Just like in $\Gamma$, a point in $\bar{\Gamma}$ is on five lines and a line has three points.) This allows us to show that $k<7$ is impossible. Indeed, suppose $k \leq 6$. Then Lemmas 2.2 and 2.1 imply that $k=6$ and $\bar{\Gamma}$ is a generalized 6-gon. However, in that case the 8-paths in $\bar{\Gamma}$ that start from a point split into two types: the end point of the path can be at distance four or six from the starting point. This contradicts the transitivity of $\bar{G}$ on 8-paths starting from a point.

Thus, $k=7$. Here again we obtain a contradiction, even if in a slightly different way. First of all, the argument in the previous paragraph implies that the girth of $\bar{\Gamma}$ has to be exactly 14 . Since $\bar{G}$ is transitive on 8-paths starting from a point, every such 8-path is contained in a 14-cycle. This implies that the maximal distance in $\bar{\Gamma}$ from a point is seven, leading to the following distance distribution diagram from a point:

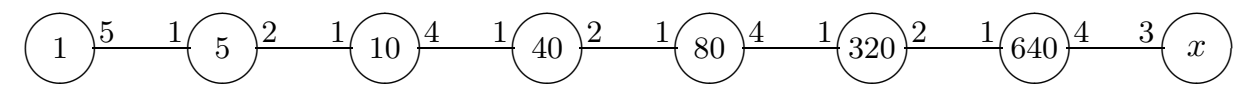

Here $x$, the number of lines at distance seven, can be found as $\frac{640 \cdot 4}{3}$, which is not an integer. This contradiction establishes that $k=8$, thus completing the proof of Theorem 3 .

\section{REFERENCES}

[DGS] A. Delgado, D. Goldschmidt and B. Stellmacher, Groups and Graphs: New Results and Methods, Birkhäuser, 1985. MR 88a:05076

[vM] H. van Maldeghem, Generalized Polygons, Birkhäuser, 1998. CMP 2000:05

[S] J.-P. Serre, Trees, Springer, 1980. MR 82c:20083

[Sz] G. Seitz, Flag-transitive subgroups of Chevalley groups, Ann. Math. 97 (1973), 27-56. MR 49:5201

[T] J. Tits, Ensembles ordonnes, immeubles at sommes amalgamees, Bull. Soc. Math. Belg. A38 (1986), 367-387. MR 88j:20041

Department of Mathematics and Statistics, Bowling Green State University, BowlING Green, OHIO 43403

E-mail address: cbennet@bgnet.bgsu.edu

Department of Mathematics and Statistics, Bowling Green State University, BowlIng Green, Оhio 43403

E-mail address: sergey@bayes.bgsu.edu 